Pacific Journal of Mathematics

aNIMAL COVERINGS OF PAIRS B B TRIP 


\title{
MINIMAL COVERINGS OF PAIRS BY TRIPLES
}

\author{
M. K. Fort, JR. AND G. A. Hedlund
}

1. Introduction. Let $F$ be a finite set with $n$ members, $n \geqq 3$. An $F$-covering of pairs by triples, which we abbreviate $F$-copt, is a set $S$ of triples of distinct members of $F$ which has the property that each pair of distinct members of $F$ is contained in at least one member of $S$. If $n$ is a positive integer, $n \geqq 3$, then an $n$-copt is an $F$-copt for the set $F=\{1,2, \cdots, n\}$. We assume throughout that $n \geqq 3$.

For any finite set $A$, let $C(A)$ denote the number of members of $A$. An $F$-copt $S$ is minimal if $C(S) \leqq C\left(S^{\prime}\right)$ for every $F$-copt $S^{\prime}$. If $n \equiv 1$ $(\bmod 6)$ or $n \equiv 3(\bmod 6)$, then a minimal $n$-copt $S$ turns out to be exact in the sense that each pair is contained in exactly one member of $S$. Such exact coverings are called Steiner triple systems. The existence of Steiner triple systems for all $n$ (of form $6 h+1$ or $6 h+3$ ) was proved by M. Reiss [2] in 1859 .

Let $S$ be a minimal $n$-copt and let $C(S)=\mu(n)$. The main result of this paper is obtained in $\S 2$, where we determine $\mu(n)$ explicitly for $n \geqq 3$. In $\S 3$ we discuss certain properties of minimal $n$-copts, and give several methods for constructing minimal $n$-copts.

2. Determination of $\mu(n)$. Let $S$ be a minimal $n$-copt. For each integer $i, 1 \leqq i \leqq n$, we define $\alpha(i)$ to be the number of members of $S$ that contain $i$. Then

$$
\sum_{i=1}^{n} \alpha(i)=3 \cdot C(S) .
$$

Since $i$ must appear in members of $S$ with $n-1$ other numbers we have $\alpha(i) \geqq[n / 2]$. ([x] is the largest integer which is not greater than $x$.) Thus,

$$
\mu(n)=C(S) \geqq \frac{n}{3}\left[\frac{n}{2}\right] .
$$

Since $(n / 3)[n / 2]$ may not be an integer, we define $\varphi(n)$ to be the least integer which is not less than $(n / 3)[n / 2]$. It is easy to compute

$$
\varphi(n)=\left\{\begin{array}{l}
n^{2} / 6 \text { if } n=6 k, \\
n(n-1) / 6 \text { if } n=6 k+1 \text { or } n=6 k+3, \\
\left(n^{2}+2\right) / 6 \text { if } n=6 k+2 \text { or } n=6 k+4, \\
\left(n^{2}-n+4\right) / 6 \text { if } n=6 k+5 .
\end{array}\right.
$$

Received May 26, 1958. The preparation of this paper was sponsored in part by the Office of Naval Research. Reproduction in whole or in part is permitted for any purpose of the United States Government. 
We may clearly improve (1) to

$$
\mu(n)=C(S) \geqq \varphi(n) .
$$

Our main theorem proves that in (3) equality holds for every $n$.

Let $A, B$ and $C$ be pairwise disjoint sets, each having the same number $n$ of members. A tricover for the system $(A, B, C)$ is a set $K$ of triples $(x, y, z), x \in A, y \in B, z \in C$ such that each pair $u v, u$ and $v$ in different ones of $A, B, C$, is contained in exactly one member of $K$.

Lemma 1. If $n$ is a positive integer and $A, B, C$ are pairwise disjoint sets each of which has $n$ members, then a tricover $K$ for $(A, B, C)$ exists. Moreover, if $a \in A, b \in B$ and $c \in C$, then $K$ may be chosen so that $(a, b, c) \in K$.

Proof. Let the members of $A, B, C$ be respectively

$$
a_{1}, a_{2}, \cdots, a_{n} ; \quad b_{1}, b_{2}, \cdots, b_{n} ; \quad c_{1}, c_{2} \cdots, c_{n},
$$

where $a_{1}=a, b_{1}=b, c_{1}=c$. We define $K$ to be the set of all triples $\left(a_{i}, b_{j}, c_{k}\right)$ for which $k \equiv i+j-1(\bmod n), 1 \leqq i, j, k \leqq n$. The set $K$ obviously has the desired properties.

REMARK. Any tricover for $(A, B, C)$ must have $n^{2}$ members.

Lemma 2. Let $A, B, C$ be pairwise disjoint sets, each having $n$ members. Let $p$ be an integer such that $0<p \leqq n / 2$. Let $A^{*} \subset A, B^{*} \subset B$, $C^{*} \subset C$ be sets, each of which has $p$ members and let $K^{*}$ be a tricover for $\left(A^{*}, B^{*}, C^{*}\right)$. Then there exists a tricover $K$ for $(A, B, C)$ such that $K^{*} \subset K$.

Proof. Let

$$
\begin{aligned}
& A=\left\{a_{1}, a_{2}, \cdots, a_{n}\right\}, \\
& B=\left\{b_{1}, b_{2}, \cdots, b_{n}\right\}, \\
& C=\left\{c_{1}, c_{2}, \cdots, c_{n}\right\} .
\end{aligned}
$$

We can assume that

$$
\begin{aligned}
& A^{*}=\left\{a_{1}, a_{2}, \cdots, a_{p}\right\}, \\
& B^{*}=\left\{b_{1}, b_{2}, \cdots, b_{p}\right\}, \\
& C^{*}=\left\{c_{1}, c_{2}, \cdots, c_{p}\right\} .
\end{aligned}
$$

For $1 \leqq i, j \leqq p$, let $m_{i j}^{*}$ be the unique integer $k$ such that $\left(a_{i}, b_{j}, c_{k}\right) \in K^{*}$. Clearly $1 \leqq m_{i j}^{*} \leqq p$ and the square array $\left(m_{i j}^{*}\right)$ is a Latin square of order $p$. It follows from a theorem of Marshall Hall [1] that there exists a Latin square $\left(m_{i j}\right), 1 \leqq i, j \leqq n$, such that $m_{i j}=m_{i j}^{*}$, 
$1 \leqq i, j \leqq p . \quad$ Let

$$
K=\left\{\left(a_{i}, b_{j}, c_{m_{i j}}\right) \mid 1 \leqq i, j \leqq n\right\} .
$$

The set $K$ is the desired tricover.

In order to produce an inductive proof of our main theorem, it is convenient to restrict ourselves to a special type of minimal $n$-copt for the case $n \equiv 5(\bmod 6)$. Also, for $n \equiv 3(\bmod 6)$, there is a special type of minimal $n$-copt whose existence we wish to establish, and it is possible to include this result in our main theorem. For these reasons we introduce the notion of "admissible $F$-copt."

An $F$-copt $S$ is admissible if $C(S)=\varphi(n), n=C(F)$, and :

(1) $n \equiv 0,1,2$, or $4(\bmod 6)$;

(2) $n \equiv 3(\bmod 6)$ and $S$ contains a set of pairwise disjoint triples whose union is $F$; or

(3) $n \equiv 5(\bmod 6)$ and $S$ contains four elements of the form $(a, b, x)$, $(a, b, y),(a, b, z),(x, y, z)$.

THEOREM 1. If $n$ is a positive integer, $n \geqq 3$, then there exists an admissible n-copt.

Proof. Our proof is by induction on $n$. However, it is necessary to prove independently that there are admissible $n$-copts for $n=3,5,7,9,11,13$, and 15 . We accomplish this by exhibiting such admissible $n$-copts.

\begin{tabular}{|c|c|c|c|c|}
\hline$n=3$ & \multicolumn{2}{|c|}{$n=9$} & \multicolumn{2}{|c|}{$n=13$} \\
\hline \multirow[t]{3}{*}{$(1,2,3)$} & $(1,2,3)$ & $(2,4,9)$ & $(1,2,3)$ & $(3,6,12)$ \\
\hline & $|(4,5,6)|$ & $(2,5,8)$ & $(1,4,5)$ & $(3,8,13)$ \\
\hline & $(7,8,9)$ & $(2,6,7)$ & $(1,6,13)$ & $(3,9,10)$ \\
\hline$n=5$ & $(1,4,7)$ & $(3,4,8)$ & $(1,7,8)$ & $(4,6,7)$ \\
\hline$(1,2,3)$ & $(1,5,9)$ & $(3,5,7)$ & $(1,9,12)$ & $(4,8,9)$ \\
\hline$(1,2,4)$ & $(1,6,8)$ & $(3,6,9)$ & $(1,10,11)$ & $(4,12,13)$ \\
\hline$(1,2,5)$ & & & $(2,4,10)$ & $(5,8,11)$ \\
\hline \multirow[t]{2}{*}{$(3,4,5)$} & \multirow{2}{*}{\multicolumn{2}{|c|}{$n=11$}} & $(2,5,6)$ & $(5,9,13)$ \\
\hline & & & $(2,7,9)$ & $(5,10,12)$ \\
\hline \multirow[b]{2}{*}{$n=7$} & $\overline{(1,2,3)}$ & $(3,6,10)$ & $(2.8,12)$ & $(6,8,10)$ \\
\hline & $(1,2,4)$ & $(3,7,9)$ & $(2,11,13)$ & $(6,9,11)$ \\
\hline$(1,2,3)$ & $(1,2,5)$ & $(3,8,11)$ & $(3,4,11)$ & $(7,10,13)$ \\
\hline$(1,4,5)$ & $(3,4,5)$ & $(4,6,11)$ & $(3,5,7)$ & $(7,11,12)$ \\
\hline$(1,6,7)$ & $(1,6,7)$ & $(4,7,8)$ & & \\
\hline$(2,4,6)$ & $(1,8,9)$ & $(4,9,10)$ & & \\
\hline$(2,5,7)$ & $(1,10,11)$ & $(5,6,9)$ & & \\
\hline$(3,4,7)$ & $(2,6,8)$ & $(5,7,11)$ & & \\
\hline \multirow[t]{2}{*}{$(3,5,6)$} & $(2,7,10)$ & $(5,8,10)$ & & \\
\hline & $(2,9,11)$ & & & \\
\hline
\end{tabular}


$n=15$

$\begin{array}{llll}(1,2,3) & (2,6,8) & (3,12,14) & (6,9,14) \\ (1,4,14) & (2,7,14) & (4,5,6) & (6,12,13) \\ (1,5,9) & (2,9,11) & (4,8,13) & \underline{(7,8,9)} \\ (1,6,10) & (2,10,15) & (4,9,10) & (7,10,13) \\ (1,7,12) & (3,4,7) & (4,11,15) & (8,11,14) \\ (1,8,15) & (3,5,11) & (5,7,15) & (9,12,15) \\ (1,11,13) & (3,6,15) & (5,8,12) & (10,11,12) \\ (2,4,12) & (3,8,10) & (5,10,14) & (13,14,15) \\ (2,5,13) & (3,9,13) & (6,7.11) & \end{array}$

Our proof now divides into six cases. In Case $r, 0 \leqq r \leqq 5$, we assume that $n \equiv r(\bmod 6)$, that $n>3$ and that there exist admissible $m$-copts for $3 \leqq m<n$. We then show that these assumptions imply that there exists an admissible $n$-copt.

Case 0. Let $S_{1}$ be an admissible $(n-1)$-copt having $(1,2,3),(1,2,4)$, and $(1,2,5)$ as three of its members. If we delete $(1,2,3)$ from $S_{1}$ and add

$$
(1,3, n),(2,3, n),(4,5, n),(6,7, n), \cdots,(n-2, n-1, n),
$$

we obtain a set $S$ of triples which is an $n$-copt. Since $S_{1}$ has

$$
\left[(n-1)^{2}-(n-1)+4\right] / 6=\left(n^{2}-3 n+6\right) / 6
$$

members, $S$ has

members.

$$
\left(n^{2}-3 n+6\right) / 6-1+n / 2=n^{2} / 6=\varphi(n)
$$

Case 1. We have exhibited admissible $n$-copts for $n=7$ and $n=13$. Therefore we may assume $n=6 h+1, h>2$.

We consider two subcases.

Subcase i. Either $h \equiv 0$ or $\mathrm{h} \equiv 1(\bmod 3)$. Then there exists $k$ such that $2 h+1=6 k+1$ or $2 h+1=6 k+3$.

Let

$$
\begin{aligned}
& A_{1}=\{1, \cdots, 2 h, n\} \\
& A_{2}=\{2 h+1, \cdots, 4 h, n\} \\
& A_{3}=\{4 h+1, \cdots, 6 h, n\}
\end{aligned}
$$

and let $S_{j}$ be an admissible $A_{j}$-copt for $j=1,2,3$. Let $T$ be a tricover for $(\{1, \cdots, 2 h\},\{2 h+1, \cdots, 4 h\},\{4 h+1, \cdots, 6 h\})$. We now define $S=S_{1} \cup S_{2} \cup S_{3} \cup T$. It is easy to verify that $S$ is an $n$-copt, and that $S$ has

$$
3 \cdot \frac{(2 h+1) 2 h}{6}+(2 h)^{2}=\frac{n(n-1)}{6}=\varphi(n)
$$

members. 
Subcase ii. $h \equiv 2(\bmod 3)$. In this case there exists $k$ such that $2 h+1=6 k+5$. We define $A_{1}, A_{2}, A_{3}$ as above. Now, for $j=0,1,2$, we let $S_{j+1}$ be an admissible $A_{j+1}$-copt such that $S_{j+1}$ contains a subset $R_{j+1}$ whose members are:

$$
\begin{aligned}
& (2 j h+1,2 j h+2,2 j h+3) \\
& (2 j h+1,2 j h+2,2 j h+4) \\
& (2 j h+1,2 j h+2, n) \\
& (2 j h+3,2 j h+4, n) .
\end{aligned}
$$

Let $T$ be a tricover for $(\{1, \cdots, 4\},\{2 h+1, \cdots, 2 h+4\}$, $\{4 h+1, \cdots, 4 h+4\})$, and let $T^{*}$ be a tricover for $(\{1, \cdots, 2 h\}$, $\{2 h+1, \cdots, 4 h\},\{4 h+1, \cdots, 6 h\})$ that is an extension of $T$. Since $\mathrm{h} \geqq 5$, the existence of such a tricover follows from Lemma 2 . We next take an admissible copt $U$ for

$$
\{1, \cdots, 4,2 h+1, \cdots, 2 h+4,4 h+1, \cdots, 4 h+4, n\} .
$$

Finally, we define

$$
S=\left(S_{1}-R_{1}\right) \cup\left(S_{2}-R_{2}\right) \cup\left(S_{3}-R_{3}\right) \cup\left(T^{*}-T\right) \cup U .
$$

It is easy to check that $S$ is an $n$-copt. The number of member of $S$ is

$$
\begin{aligned}
3 \cdot\left[\frac{(2 h+1)^{2}-(2 h+1)+4}{6}-4\right] & +\left[(2 h)^{2}-16\right]+26 \\
& =6 h^{2}+h=\frac{n(n-1)}{6} .
\end{aligned}
$$

Thus, $S$ is admissible.

Case 2. Let $S_{1}$ be an admissible $(n-1)$-copt. We define $S$ to be the set of triples obtained by adding to $S_{1}$ the triples

$$
(1,2, n),(3,4, n), \cdots,(n-3, n-2, n),(n-2, n-1, n) .
$$

Then, $S$ is an $n$-copt and $S$ has

$$
\frac{(n-1)(n-2)}{6}+\frac{n}{2}=\frac{n^{2}+2}{6}
$$

members. Thus $S$ is admissible.

Case 3. There exists $h$ such that $n=6 h+3$. Since we have listed admissible $n$-copts for $n=3,9,15$, we may assume $h>2$. We consider two subcases. 
Subcase i. $h \equiv 0$ or $h \equiv 1(\bmod 3)$. In this case there exists $k$ such that $2 h+1=6 k+1$ or $2 h+1=6 k+3$. Let $S_{1}$ be an admissible $(2 h+1)$-copt. For each triple $(a, b, c) \in S_{1}$ we choose a tricover for $(\{3 a-2$, $3 a-1,3 a\},\{3 b-2,3 b-1,3 b\},\{3 c-2,3 c-1,3 c\})$. The union of all such tricovers, together with the triples $(1,2,3),(4,5,6), \cdots,(n-2, n-1, n)$ is an $n$-copt $S$. The number of members of $S$ is

$$
9 \cdot \frac{(2 h+1) \cdot 2 h}{6}+(2 h+1)=(2 h+1)(3 h+1)=\frac{n(n-1)}{6} .
$$

If follows that $S$ is admissible.

Subcase ii. $h \equiv 2(\bmod 3)$. In this case there exists $k$ such that $2 h+1=6 k+5$. We choose an admissible $(2 h+1)$-copt $S_{1}$ that contains the triples $(1,2,3),(1,2,4),(1,2,5),(3,4,5)$. If $(a, b, c)$ is any other member of $S_{1}$, we choose a tricover for $(\{3 \alpha-2,3 \alpha-1,3 \alpha\}$, $\{3 b-2,3 b-1,3 b\},\{3 c-2,3 c-1,3 c\})$. Let $S_{2}$ be the 15-copt exhibited at the beginning of our proof. We now define $S$ to be the set whose members are the members of $S_{2}$, the members of the chosen tricovers, and the triples $(16,17,18), \cdots,(n-2, n-1, n) . \quad S$ is an $n$-copt, and the number of members of $S$ is

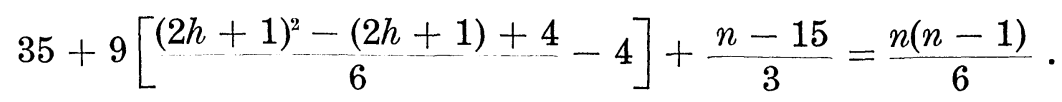

Since $S$ has $(1,2,3),(4,5,6), \cdots,(n-2, n-1, n)$ as members, $S$ is admissible.

Case 4. For this case, the construction is exactly the same as in Case 2.

Case 5. We first observe that numbers of the form $6 h+5, h$ a non-negative integer, form the same set as numbers of the form $3 s-4$, $s$ an odd integer and $s>1$. We have listed an admissible 5-copt, and an admissible 11-copt. Thus, we may assume $n=6 h+5=3 s-4, s>5$. We consider two subcases.

Subcase i. There exists $k$ such that $s=6 k+1$ or $s=6 k+3$. In this case, we let

$$
\begin{aligned}
& A_{1}=\{1, \cdots, s-2\} \\
& A_{2}=\{s-1, \cdots, 2 s-4\} \\
& A_{3}=\{2 s-3, \cdots, 3 s-6\} .
\end{aligned}
$$

There is a tricover $K$ of $\left(A_{1}, A_{2}, A_{3}\right)$ such that $(1, s-1,2 s-3) \in K$. For $i=1,2,3$ we define 


$$
R_{i}=A_{i} \cup\{3 s-5,3 s-4\} .
$$

and let $S_{i}$ be an admissible $R_{i}$-copt such that $(1,3 s-5,3 s-4) \in S_{1}$, $(s-1,3 s-5,3 s-4) \in S_{2}$ and $(2 s-3,3 s-5,3 s-4) \in S_{3}$. We define $S=K \cup S_{1} \cup S_{2} \cup S_{3}$. It is easy to see that $S$ is an $n$-copt, and that $S$ has

$$
(s-2)^{2}+\frac{3 s(s-1)}{6}=\frac{3 s^{2}-9 s+8}{2}=\frac{n^{2}-n+4}{6}
$$

members. Since $(1,3 s-5,3 s-4),(s-1,3 s-5,3 s-4),(2 s-3,3 s-5,3 s-4)$, $(1, s-1,2 s-3)$ are members of $S, S$ is admissible.

Subcase ii. There exists $k$ such that $s=6 k+5$. We define

$$
\begin{aligned}
& A_{1}=\{1, \cdots, s-2\} \\
& A_{2}=\{s-1, \cdots, 2 s-4\} \\
& A_{3}=\{2 s-3, \cdots, 3 s-6\}
\end{aligned}
$$

and let $R_{i}=A_{i} \cup\{3 s-5,3 s-4\}$ for $i=1,2,3$. By the inductive hypothesis, there exists an admissible $R_{i}$-copt $\mathrm{S}_{\imath}$ such that $S_{i}$ contains the set $B_{i}$, where

$$
\begin{gathered}
\left.B_{1}=\{1,2,3),(1,3 s-5,3 s-4),(2,3 s-5,3 s-4),(3,3 s-5,3 s-4)\right\}, \\
B_{2}=\{(s-1, s, s+1),(s-1,3 s-5,3 s-4),(s, 3 s-5,3 s-4), \\
(s+1,3 s-5,3 s-4)\} . \\
B_{3}=\{(2 s-3,2 s-2,2 s-1),(2 s-3,3 s-5,3 s-4),(2 s-2,3 s-5,3 s-4), \\
(2 s-1,3 s-5,3 s-4)\} .
\end{gathered}
$$

Let $G=\{1,2,3, s-1, s, s+1,2 s-3,2 s-2,2 s-1,3 s-5,3 s-4\}$. $G$ has 11 members, and hence there exists an admissible $G$-copt $M$.

We choose a tricover $T_{1}$ for $(\{1,2,3\},\{s-1, s, s+1\},\{2 s-3$, $2 s-2,2 s-1\})$ and extend $T_{1}$ to a tricover $T$ for $\left(A_{1}, A_{2}, A_{3}\right)$.

We now define

$$
S=\left(S_{1}-B_{1}\right) \cup\left(S_{2}-B_{2}\right) \cup\left(S_{3}-B_{3}\right) \cup M \cup\left(T-T_{1}\right) .
$$

It is a routine matter to verify that $S$ is an $n$-copt. The number of members of $S$ is

$$
3\left[\frac{s^{2}-s+4}{6}-4\right]+19+\left[(s-2)^{2}-9\right]=\frac{3 s^{2}-9 s+8}{2}=\frac{n^{2}-n+4}{6} .
$$

Since $S \supset M$ and $M$ is admissible, it follows that $S$ is admissible.

3. Properties of minimal $n$-copts. Let $S$ be a minimal $n$-copt. If $n \equiv r(\bmod 6)$, for $r=0,2,4,5$, then the covering is not exact and some 
pairs must be contained in more than one member of $S$. However, it is possible to state precisely the way in which this sort of "multiple covering" takes place. Our results are contained in the next three theorems.

THEOREM 2. Let $n=6 k$, and let $S$ be an $n$-copt for which $C(S)=\varphi(n)$. There exists a partition of $\{1,2, \cdots, n\}$ into $3 k$ pairs $P_{1}, P_{2}, \cdots, P_{3 k}$, each of which is contained in exactly two members of $S$. Every other pair $(u, v), 1 \leqq u<v \leqq n$, is contained in exactly one member of $S$.

Proof. For $1 \leqq j \leqq n$, let $f(j)$ be the number of members of $S$ that contain $j$. It is clear that $f(j)$ is at least $n / 2$, so that $f(j)=n / 2+g(j)$, $g(j) \geqq 0$. We obtain

$$
\sum_{j=1}^{n} f(j)=3 \varphi(n)
$$

Thus

$$
\begin{gathered}
\sum_{j=1}^{n}\left[\frac{n}{2}+g(j)\right]=3 \cdot \frac{n^{3}}{6}, \text { and } \\
\frac{n^{2}}{2}+\sum_{j=1}^{n} g(j)=\frac{n^{2}}{2} .
\end{gathered}
$$

We see that $g(j)=0$ for $j=1, \cdots, n$ and $f(j)=n / 2$. Since for each $k \neq j$ there is at least one member of $S$ which contains $(j, k)$, there must exist $j^{*} \neq j$ such that $\left(j, j^{*}\right)$ is contained in exactly two members of $S$, and $(j, k)$ is contained in exactly one member of $S$ for $j \neq k \neq j^{*}$. Moreover, $j^{* *}=j$, and hence the pairs $\left(j, j^{*}\right)$ are the $n / 2$ pairs $P_{1}, P_{2}, \cdots, P_{3 k}$.

THEOREM 3. Let $n=6 k+2$ or $n=6 k+4$, and let $S$ be an $n$-copt for which $C(S)=\varphi(n)$. There exist $n / 2+1$ pairs $P_{1}, \cdots, P_{n / 2+1}$ which are contained in exactly two members of $S$. Every other pair is contained in exactly one momber of $S$. Thore exists wir integer m which is contained in exactly three of the pairs $P_{1}, \cdots, P_{n / 2+1}$. Every other integer is contained in exactly one of the pairs $P_{1}, \cdots, P_{n / 2+1}$.

Proof. Let $f(j)$ be the number of members of $S$ that contain the integer $j$. Since $f(j) \geqq n / 2$, we can write

$$
f(j)=\frac{n}{2}+g(j), \quad g(j) \geqq 0 .
$$

Then 


$$
\sum_{j=1}^{n} f(j)=\frac{n^{2}}{2}+\sum_{j=1}^{n} g(j)=3 \cdot \varphi(n)=\frac{n^{2}+2}{2} .
$$

Thus $\sum_{j=1}^{n} g(j)=1$. There exists an integer $m$ such that $g(m)=1$ and $g(j)=0$ for $j \neq m$.

Now suppose $j \neq m$. There must exist $j^{*}$ such that $\left(j, j^{*}\right)$ is contained in exactly two members of $S$, and $(j, h)$ is contained in exactly one member of $S$ for $j \neq h \neq j^{*}$.

Since there are $n / 2+1$ members of $S$ that contain $m$, and each pair $(m, j)$ is contained in at least one and not more than two members of $S$, there exist $a, b, c$, such that $(m, a),(m, b),(m, c)$ are each contained in exactly two members of $S$, and $(m, j)$ is contained in exactly one member of $S$ if $j \neq a, j \neq b$, and $j \neq c$.

If $j$ is a member of $T=\{1, \cdots, n\}-\{m, a, b, c\}$, then $j^{* *}=j$. Hence $T$ is partitioned into pairs $P_{1}, P_{2}, \cdots, P_{(n-4) / 2}$, each of which is contained in exactly two members of $S$. These pairs, together with $(m, a),(m, b),(m, c)$ form the set $P_{1}, \cdots, P_{n / 2+1}$.

THEOREM 4. If $n=6 k+5$ and $S$ is a minimal n-copt for which $\varphi(n)=\left(n^{2}-n+4\right) / 6$, then one pair is contained in three members of $S$ and every other pair is contained in exactly one member of $S$.

Proof. For $1 \leqq j \leqq n$, we define $f(j)$ to be the number of members of $S$ that contain $j$. Clearly $f(j) \geqq(n-1) / 2$. We define $g(j)=f(j)-$ $(n-1) / 2$. Since $\sum_{j=1}^{n} f(j)=3 \varphi(n)=\left(n^{2}-n+4\right) / 2$, we obtain

$$
\sum_{j=1}^{n} g(j)=2 \text {. }
$$

There exists $j_{1}$ such that $g\left(j_{1}\right)>0$. Since there are more than $(n-1) / 2$ triples of $S$ that contain $j_{1}$, there exists $j_{2}$ such that the pair $\left(j_{1}, j_{2}\right)$ is contained in at least two triples $\left(j_{1}, j_{2}, j_{3}\right),\left(j_{1}, j_{2}, j_{4}\right)$. The integer $j_{2}$ must be in triples with $n-4$ integers other than $j_{1}, j_{3}, j_{4}$, and it requires at least $(n-3) / 2$ triples to satisfy this condition. Thus $f\left(j_{2}\right) \geqq(n+1) / 2$ and $g\left(j_{2}\right)>0$. We now see that $g\left(j_{1}\right)=g\left(j_{2}\right)=1$ and $g(j)=0$ if $j_{1} \neq j \neq j_{2}$.

It now follows that if $(u, v)$ is a pair for which $g(u)=0$ or $g(v)=0$, then $(u, v)$ is contained in exactly one member of $S$. Since $3 \varphi(n)=$ $n(n-1) / 2+2$, the pair $\left(j_{1}, j_{2}\right)$ must be contained in three members of $S$.

Our Theorem 1 is of a constructive nature, and indicates how minimal $n$-copts can be constructed out of minimal $m$-copts for $m<n$. There are other methods, however, of constructing minimal $n$-copts out of minimal $m$-copts for $m<\mathrm{n}$. We give a lemma and theorem due to Reiss [2] which are useful in this connection. Our final theorem is analogous to the Reiss Theorem. 
ReISS Lemma. Let $n$ be a positive integer. Let

$$
P=\{(u, v) \mid 1 \leqq u<v \leqq 2 n\} .
$$

Then there exists a partition of $P$ into sets $S_{1}, S_{2}, \cdots, S_{2 n-1}$, each containing $n$ elements, such that for each $i, i=1,2, \cdots, 2 n-1$, the coordinates of the $n$ pairs in $S_{i}$ constitute the integers $1,2, \cdots, 2 n$.

Proof. Let $j$ be an integer such that $1 \leqq j \leqq 2 n-1$. We define

$$
T_{\jmath}=\{(a, b) \mid 1 \leqq a<b \leqq j+1 \text { and } a+b=j+2\}
$$

and

$$
R_{j}=\{(a, b) \mid j+1<a<b<2 n \text { and } a+b=j+2 n+1\} .
$$

Let $S_{2 n-1}=T_{2 n-1}$. For $j$ even, $1 \leqq j \leqq 2 n-2$, let

$$
S_{\jmath}=T_{j} \cup R_{\jmath} \cup\left\{\left(\frac{j+2}{2}, 2 n\right)\right\} \text {. }
$$

For $j$ odd, $1 \leqq j \leqq 2 n-3$, let

$$
S_{j}=T_{j} \cup R_{j} \cup\left\{\left(\frac{j+1+2 n}{2}, 2 n\right)\right\} .
$$

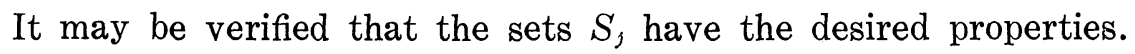

Reiss TheOREM. Let $m$ be odd and let $S$ be an $m$-copt for which $C(S)=\varphi(m)$. Then there exists a $(2 m+1)$-copt $T$ such that $T \supset S$ and $C(T)=\varphi(2 m+1)$.

Proof. Let $P=\{(u, v) \mid m<u<v \leqq 2 m+1\}$. We use the Reiss lemma to partition $P$ into sets $S_{1}, \cdots, S_{m}$, each containing $(m+1) / 2$ elements, such that for each $i, i=1,2, \cdots, m$, the coordinates of the $(m+1) / 2$ pairs in $S_{i}$ constitute the integers $m+1, m+2, \cdots, 2 m+1$. We now define

$$
T=S \cup\left\{(i, j, k) \mid 1 \leqq i \leqq m \text { and }(j, k) \in S_{i}\right\} .
$$

It is easily verified that $T$ is a $(2 m+1)$-copt. If $m \equiv 1$ or $m \equiv 3(\bmod 6)$, then

$$
C(S)=\frac{m(m-1)}{6}+\frac{m(m+1)}{2}=\frac{4 m^{2}+2 m}{6}=\frac{(2 m+1)(2 m)}{6}=\varphi(2 m+1) .
$$

If $m \equiv 5(\bmod 6)$, then

$$
\begin{aligned}
C(S) & =\frac{m^{2}-m+1}{6}+\frac{m(m+1)}{2}=\frac{4 m^{2}+2 m+4}{6} \\
& =\frac{(2 m+1)^{2}-(2 m+1)+4}{6}=\varphi(2 m+1) .
\end{aligned}
$$


THEOREM 5. Let $n$ be an even integer and let $S$ be an $n$-copt for which $C(S)=\varphi(n)$. Then there exists a $2 n$-copt $T$ such that $C(T)=\varphi(2 n)$ and $S \subset T$.

Proof. According to the Reiss Lemma there exists a partition of the set

$$
P=\{(u, v) \mid n+1 \leqq u<v \leqq 2 n\}
$$

into $n-1$ sets $A_{1}, A_{2}, \cdots, A_{n-1}$ such that for each $i, i=1,2, \cdots, n-1$, the coordinates of the $n / 2$ pairs in $A_{i}$ constitute the integers $\{n+1, \cdots, 2 n\}$. Let $A_{n}=A_{n-1}$, and let

$$
T=S \cup\left\{(i, j, k) \mid i=1,2, \cdots, n ;(j, k) \in A_{i}\right\} .
$$

It is easy to prove that $T$ satisfies the desired conditions.

\section{REFERENCES}

1. Marshall Hall, An exislence theorem for Latin squares, Bull. Amer. Math Soc. 51 (1945), 387.

2. M. Reiss, Ueber eine Sieinersche combinatorische Aufgabe welche im 45 sten Bande dieses Journal, Seite 181, gestellt worden ist. J. reine angew. Math. 56 (1859), 326-344.

UNIVERSITY OF GeORGIA AND YALE UNIVERSITY 



\section{PACIFIC JOURNAL OF MATHEMATICS}

\section{EDITORS}

\section{David Gilbarg}

Stanford University

Stanford, California

\section{R. A. Beaumont}

University of Washington

Seattle 5, Washington

\author{
A. L. Whiteman
}

University of Southern California Los Angeles 7, California

E. G. Straus

University of California

Los Angeles 24, California

\section{ASSOCIATE EDITORS}

\author{
E. F. BECKENBACH \\ C. E. BURGESS \\ M. HALL \\ E. HEWITT
}
A. HORN
V. GANAPATHY IYER
R. D. JAMES
M. S. KNEBELMAN
L. NACHBIN
I. NIVEN
T. G. OSTROM
H. L. ROYDEN

M. M. SCHIFFER

G. SZEKERES

F. WOLF

K. YOSIDA

\section{SUPPORTING INSTITUTIONS}

\author{
UNIVERSITY OF BRITISH COLUMBIA \\ CALIFORNIA INSTITUTE OF TECHNOLOGY \\ UNIVERSITY OF CALIFORNIA \\ MONTANA STATE UNIVERSITY \\ UNIVERSITY OF NEVADA \\ OREGON STATE COLLEGE \\ UNIVERSITY OF OREGON \\ OSAKA UNIVERSITY \\ UNIVERSITY OF SOUTHERN CALIFORNIA
}

\author{
STANFORD UNIVERSITY \\ UNIVERSITY OF TOKYO \\ UNIVERSITY OF UTAH \\ WASHINGTON STATE COLLEGE \\ UNIVERSITY OF WASHINGTON \\ * * * * \\ AMERICAN MATHEMATICAL SOCIETY \\ CALIFORNIA RESEARCH CORPORATION \\ HUGHES AIRCRAFT COMPANY \\ THE RAMO-WOOLDRIDGE CORPORATION
}

Mathematical papers intended for publication in the Pacific Journal of Mathematics should be typewritten (double spaced), and the author should keep a complete copy. Manuscripts may be sent to any one of the four editors. All other communications to the editors should be addressed to the managing editor, E. G. Straus at the University of California, Los Angeles 24, California.

50 reprints per author of each article are furnished free of charge; additional copies may be obtained at cost in multiples of 50 .

The Pacific Journal of Mathematics is published quarterly, in March, June, September, and December. The price per volume (4 numbers) is $\$ 12.00$; single issues, $\$ 3.50$. Back numbers are available. Special price to individual faculty members of supporting institutions and to individual members of the American Mathematical Society: $\$ 4.00$ per volume; single issues, $\$ 1.25$.

Subscriptions, orders for back numbers, and changes of address should be sent to Pacific Journal of Mathematics, 2120 Oxford Street, Berkeley 4, California.

Printed at Kokusai Bunken Insatsusha (International Academic Printing Co., Ltd.), No. 6, 2-chome, Fujimi-cho, Chiyoda-ku, Tokyo, Japan.

PUBLISHED BY PACIFIC JOURNAL OF MATHEMATICS, A NON-PROFIT CORPORATION

The Supporting Institutions listed above contribute to the cost of publication of this Journal, but they are not owners or publishers and have no responsibility for its content or policies. 


\section{Pacific Journal of Mathematics}

\section{Vol. 8, No. $4 \quad$ June, 1958}

Richard Arens, The maximal ideals of certain functions algebras ........ 641

Glen Earl Baxter, An operator identity ........................... 649

Robert James Blattner, Automorphic group representations ........... 665

Steve Jerome Bryant, Isomorphism order for Abelian groups ............ 679

Charles W. Curtis, Modules whose annihilators are direct summands...... 685

Wilbur Eugene Deskins, On the radical of a group algebra ............ 693

Jacob Feldman, Equivalence and perpendicularity of Gaussian

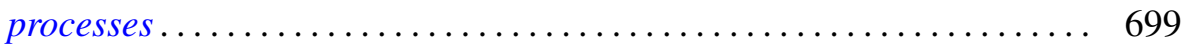

Marion K. Fort, Jr. and G. A. Hedlund, Minimal coverings of pairs by triples....................................... 709

I. S. Gál, On the theory of $(m, n)$-compact topological spaces ......... 721

David Gale and Oliver Gross, A note on polynomial and separable games........................................ 735

Frank Harary, On the number of bi-colored graphs ............... 743

Bruno Harris, Centralizers in Jordan algebras ................... 757

Martin Jurchescu, Modulus of a boundary component ............... 791

Hewitt Kenyon and A. P. Morse, Runs . . . . . . . . . . . . . . . . . . . . . . 811

Burnett C. Meyer and H. D. Sprinkle, Two nonseparable complete metric

spaces defined on $[0,1] \ldots \ldots \ldots \ldots \ldots \ldots \ldots \ldots \ldots \ldots \ldots . \ldots . \ldots . \ldots . \ldots 25$

M. S. Robertson, Cesàro partial sums of harmonic series expansions...... 829

John L. Selfridge and Ernst Gabor Straus, On the determination of numbers by their sums of a fixed order ........................ 847

Annette Sinclair, A general solution for a class of approximation

problems .................................

George Szekeres and Amnon Jakimovski, $(C, \infty)$ and $(H, \infty)$ methods of summation...................................... 867

Hale Trotter, Approximation of semi-groups of operators. ............. 887

L. E. Ward, A fixed point theorem for multi-valued functions ........... 921

Roy Edwin Wild, On the number of lattice points in $x^{t}+y^{t}=n^{t / 2} \ldots \ldots .929$ 